\title{
Graphene-enabled electrically controlled terahertz spatial light modulators
}

\author{
Nurbek Kakenov, ${ }^{1}$ Taylan Takan, ${ }^{2}$ Vedat Ali Ozkan, ${ }^{2}$ Osman Balcı, ${ }^{1}$ Emre O. Polat, ${ }^{1}$ \\ Hakan Altan, ${ }^{2,3}$ and Coskun Kocabas ${ }^{1, *}$ \\ ${ }^{1}$ Bilkent University, Department of Physics, 06800 Ankara, Turkey \\ ${ }^{2}$ Middle East Technical University, Department of Physics, Ankara, Turkey \\ ${ }^{3}$ e-mail: haltan@metu.edu.tr \\ *Corresponding author: ckocabas@fen.bilkent.edu.tr
}

Received January 29, 2015; revised March 31, 2015; accepted April 2, 2015; posted April 2, 2015 (Doc. ID 233202); published April 22, 2015

\begin{abstract}
In this Letter, we demonstrate a broadband terahertz (THz) spatial light modulator using $5 \times 5$ arrays of large area graphene supercapacitors. Our approach relies on controlling spatial charge distribution on a passive matrix array of patterned graphene electrodes. By changing the voltage bias applied to the rows and columns, we were able to pattern the THz transmittance through the device with high modulation depth and low operation voltage. We anticipate that the simplicity of the device architecture with high contrast THz modulation over a broad spectral range could provide new tools for THz imaging and communication systems. (c) 2015 Optical Society of America OCIS codes: (160.4236) Nanomaterials; (110.6795) Terahertz imaging; (130.4110) Modulators; (130.0250) Optoelectronics.

http://dx.doi.org/10.1364/OL.40.001984
\end{abstract}

Due to the nonionizing nature and submillimeter resolution of terahertz $(\mathrm{THz})$ radiation, it promises to enable new technologies ranging from homeland security to biomedical applications [1-3]. Although THz sources and detectors have significantly advanced, the active $\mathrm{THz}$ components are still underdeveloped due to the lack of an active material that works in $\mathrm{THz}$ frequencies [1]. The quest for active $\mathrm{THz}$ devices has led researchers to use high mobility free carriers to manipulate light-matter interactions in the $\mathrm{THz}$ regime $[1,4]$. Active devices such as modulators [5] and phase shifters [6,7] have been demonstrated by tuning the charge density on semiconductor surfaces. Spatial light modulators (SLMs) are the key components for beam steering and imaging systems, which involve sophisticated integration of many active components [-13]. A THz SLM requires individually addressable pixels that independently control the transmittance and reflectance of $\mathrm{THz}$ signals at different locations. Chan et al. used an interconnected $\mathrm{THz}$ metamaterial on a semiconductor surface to form Schottky contacts [5]. By wiring the group of split-ring resonators, the local transmittance of the THz waves was controlled by electrically tuning the depletion area under the Schottky contacts [6,8]. More recently, Watts et al. applied the same principle for compressive $\mathrm{THz}$ imaging [14]. Other approaches such as microelectromechanical systems and photo-generated carriers on semiconductors have also been investigated $[10,15,16]$.

The ability to synthesize large scale graphene, twodimensional (2D) crystal of carbon [17], provides a new foundation for the development of active THz devices [18-21]. The linear band structure, together with the atomic thickness of graphene, yield a high mobility 2D electron gas with gate-tunable charge density. Unlike other buried 2D electronic systems based on semiconductor heterostructures [22], graphene can be transfer-printed on an open dielectric surface that can be electrostatically doped with a back-gate electrode. In pioneering work by Sensale-Rodriguez et al. [23], a back-gated transistor was used to control transmission of THz waves. Shifting the Fermi energy of graphene changes the rate of intraband electronic transitions that alters the absorption and reflection of $\mathrm{THz}$ waves in broad spectra. The researchers extended their technique by fabricating arrays of transistors that operate as a $\mathrm{THz}$ SLM [24-30]. However, the dielectric breakdown of a back-gated transistor limits the dynamic range of these devices due to the limited charge density on the order of $10^{12} \mathrm{~cm}^{-2}$. To solve this problem, we developed a high contrast $\mathrm{THz}$ modulator using graphene supercapacitors $[31,32]$. In our device, we used two large area graphene electrodes separated by an ionic liquid electrolyte ([deme][Tf2N]) sandwiched between two graphene electrodes. This simple device architecture results in a very efficient electrolyte gating that generates charge densities on the order of $10^{14} \mathrm{~cm}^{-2}$, which corresponds to Fermi energies of $\sim 1 \mathrm{eV}$ [31,32]. The efficient electrolyte gating is achieved by very thin (a few nanometers) electrical double layers on the graphene surface. In this Letter, we demonstrate an electrically controlled, high contrast, broadband THz SLM using arrays of graphene supercapacitors. We were able to pattern the THz transmittance through a $5 \times 5$ passive matrix array of a graphene capacitor by switching the voltages applied to the rows and columns of the array. Figure 1 shows the schematic drawing of the passive matrix array of a graphene supercapacitor that operates as a THz SLM. The voltage difference between the top and the bottom electrodes polarizes the electrolyte and accumulates high mobility charges at each pixel. By changing the bias voltages applied on rows and columns of the passive matrix array, we were able to reconfigure the $\mathrm{THz}$ transmittance. We fabricated the SLM using large area graphene $\left(5 \times 5 \mathrm{~cm}^{2}\right)$ synthesized by chemical vapor deposition on copper foils and transferred on to a THz transparent polymer substrate. After the transfer process, we patterned the graphene electrodes into ribbons with a width of $8 \mathrm{~mm}$. We attached the patterned graphene electrodes 
(a)

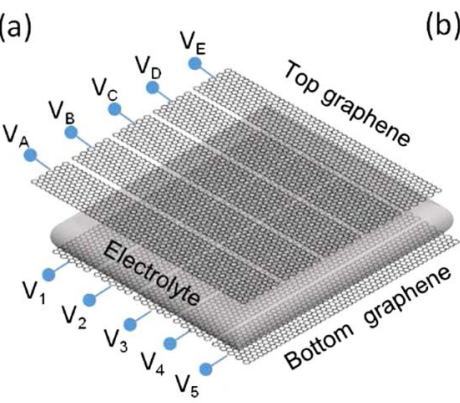

(b)

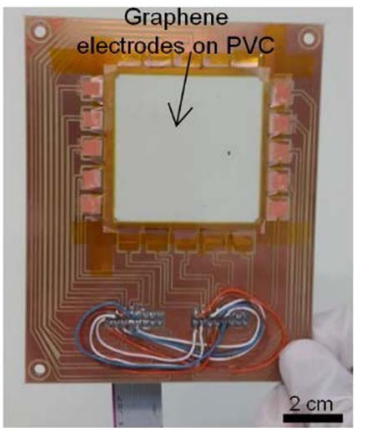

Fig. 1. (a) Schematic drawing of the THz spatial light modulator consisting of a $5 \times 5$ array of graphene supercapacitors. (b) Photograph of the fabricated device mounted on a printed circuit board. The size of an individual pixel is $8 \mathrm{~mm} \times 8 \mathrm{~mm}$.

on a printed circuit board [Fig. 1(b)] and made electrical contacts with the aid of a conductive epoxy. The intersections of rows and columns define individually addressable pixels. To address a pixel (reduce the transmittance), we apply a voltage difference between the corresponding row and column. We used a switch box and a two-channel source-measure unit (Keithley, 2600) to apply voltages to the rows and columns.

To measure a transmittance, Virginia Diode, Inc.'s Schottky-diode-based, multiplied millimeter (mm)-wave/ $\mathrm{THz}$ emitter is used as a tunable frequency signal source (WR9.0AMC+WR2.8X3). Driven by a voltage-controlled frequency-tunable YIG oscillator, the system can provide a minimum average output power of about $1 \mathrm{~mW}$. The source can operate in two frequency regions: 80-125 GHz and 240-380 GHz. The output of the source is amplitude-modulated via a function generator in order to detect the radiation with a Golay cell (Tydex, TC-1T).

The output beam was collimated with an F\#3, $D=$ $50 \mathrm{~mm}$ TPX lens and then focused onto the device with an F\#2, $D=50 \mathrm{~mm}$ Teflon lens. The mm-wave and $\mathrm{THz}$ spot diameters were measured to be $\sim 9 \mathrm{~mm}$ and $3 \mathrm{~mm}$, respectively, at the device position. The radiation was then collected by a duplicate set of optics and focused onto the receiver. The modulator was positioned in a computer-controlled $x y$-scanner (Marzhauser Wetzlar $\mathrm{GmbH}$ ), which allowed the device to be raster-scanned around the focused $\mathrm{THz}$ beam.

Figures 2(a)-2(d) show the recorded transmission images of the THz SLM with various voltage configuration at a frequency of $0.37 \mathrm{THz}$. First we measured the transmittance image when all electrodes were grounded [Fig. 2(a)]. For this configuration, the average transmittance is around $62 \pm 5 \%$. The variation $( \pm 5 \%$ of the incidence power) of the transmittance over the device area is due to inhomogeneous charge density caused by unintentional doping on graphene. When we applied +1 and $-1 \mathrm{~V}$ to the rows and columns, respectively, we observed a minimum transmittance of $30 \pm 5 \%$ [Fig. 2(b)]. To address a single pixel in a passive matrix array, one needs three distinct states. For our device, we chose 0, 1, and $2 \mathrm{~V}$ voltage differences, which yielded $65 \%, 45 \%$, and $32 \%$ of transmittance, respectively. By switching the voltages on the rows and columns, we were able to modulate the transmittance pattern. At the intersection of the grounded row and column, the voltage difference is $0 \mathrm{~V}$, which yields a high level of transmittance. However, the maximum transmittance through a single pixel depends on voltage applied to the adjacent pixel due to the cross talk. We obtained a transmittance image [the checkerboard image seen in Fig. 2(d)] with maximum and minimum transmittance of $52 \%$ and $30 \%$, respectively.
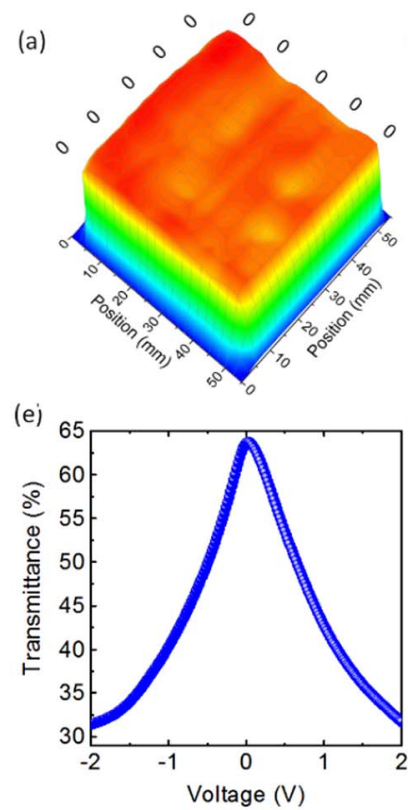
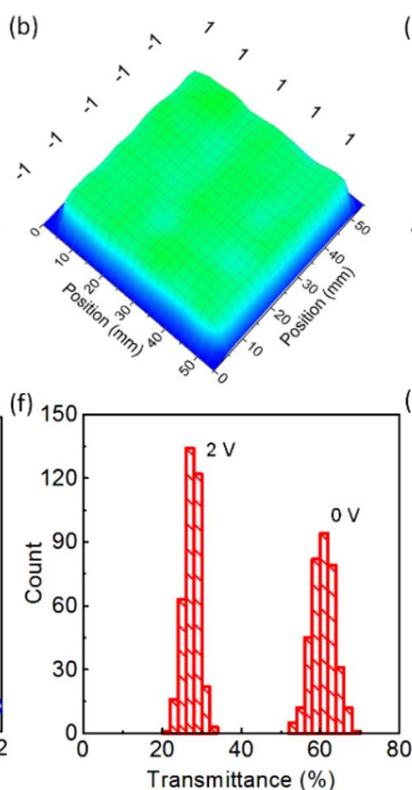

(c)

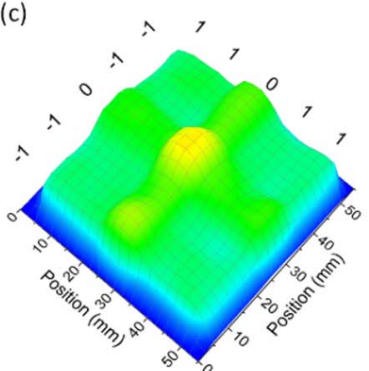

(g)

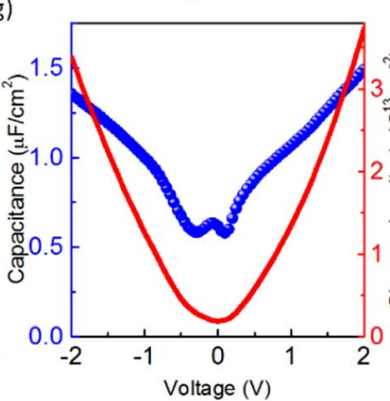

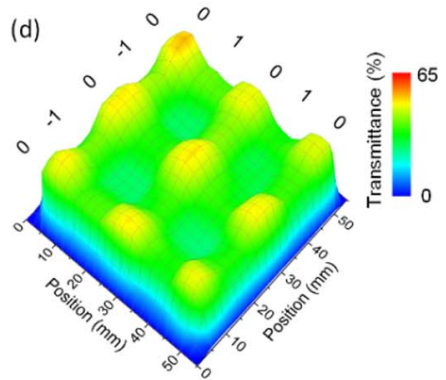

(h)

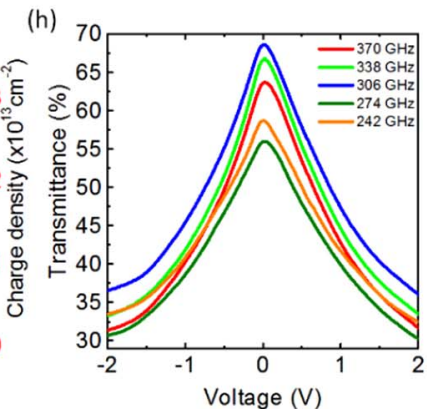

Fig. 2. (a)-(d) THz transmission images of the SLM with various voltage configurations recorded at a frequency of $0.37 \mathrm{THz}$. (e) Transmittance of the device at $0.37 \mathrm{THz}$ as a function of bias voltage. (f) Histogram of the transmittance at bias voltage of 0 and $2 \mathrm{~V}$. (g) Variation of the capacitance (left axis) of the device and estimated charge density (right axis) as a function of bias voltage. Two distinct minima are associated with the Dirac points of the top and bottom graphene electrodes. (h) Voltage-dependent transmittance at different frequencies. 
By varying the voltage from 0 to $2 \mathrm{~V}$, we can tune the transmittance from $65 \%$ down to $30 \%$ [Fig. 2(e)]. Due to the unintentional doping of graphene and hysteresis effects, there is around 5\% variation in the transmittance over the device area of $25 \mathrm{~cm} \times 25 \mathrm{~cm}$. The histogram of the transmittance at 0 and $2 \mathrm{~V}$ is given in Fig. 2(f). To quantify the amount of charge accumulated on the graphene electrodes, we measured the variation of capacitance of the device by applying a small $\mathrm{AC}$ voltage $(0.1 \mathrm{~V})$ with a variable DC bias voltage $(-2$ to $2 \mathrm{~V})$. The capacitance of the device varies from 17 to $45 \mu \mathrm{F}$. The capacitance of the device can be reconfigured by the switch box. We observed two distinct minima at $-0.3 \mathrm{~V}$ and $0.2 \mathrm{~V}$ associated with the difference of the Dirac points of the top and bottom graphene electrodes. From the measured capacitance, we estimated that the charge density varies from $0.2 \times 10^{13}$ to $3.7 \times 10^{13} \mathrm{~cm}^{-2}$. The response time of our device is slower than the dielectric based modulators due to the large capacitance and device area. The dynamic range of our modulator is limited by the minimum charge density on graphene and the electrochemical window of the electrolyte. Unintentional doping and charge puddle formation limit the minimum charge density to around $10^{12} \mathrm{~cm}^{-2}$. The electrochemical window of the electrolyte determines the maximum voltage of $4 \mathrm{~V}$, which yields a charge density of $10^{14} \mathrm{~cm}^{-2}$ [31].

An important aspect of our device is that it can operate over a very broad spectrum. We recorded the voltage dependent $\mathrm{THz}$ for a frequency range between 0.24 and $0.37 \mathrm{THz}$ [Fig. 2(h)]. This frequency range is limited by our experimental setup. The recorded transmittance varies between $67 \%$ down to $30 \%$ for this frequency range. The variation of transmittance between various frequencies over different applied potentials is most likely due to the variation of the spot size on the sample.

Since the modulation of $\mathrm{THz}$ waves is due to a changing Drude-like metallic behavior, graphene can inherently respond in a very broad spectrum. To test the broadband spectral response, we measured the spectral response using conventional time-domain $\mathrm{THz}$ spectroscopy of a single-pixel graphene device. We measured the transmitted $\mathrm{THz}$ pulse through the device at different bias voltages [Fig. 3(a)]. We took the fast Fourier transform of the time-varying electric field, calculated the transmission spectrum from 0.1 to $1.5 \mathrm{THz}$, and plotted the modulation $\left(M=\left(T_{0 \mathrm{~V}}-T_{2 \mathrm{~V}}\right) / T_{0 \mathrm{~V}}\right)$ in Fig. $\underline{3(\mathrm{~b})}$.
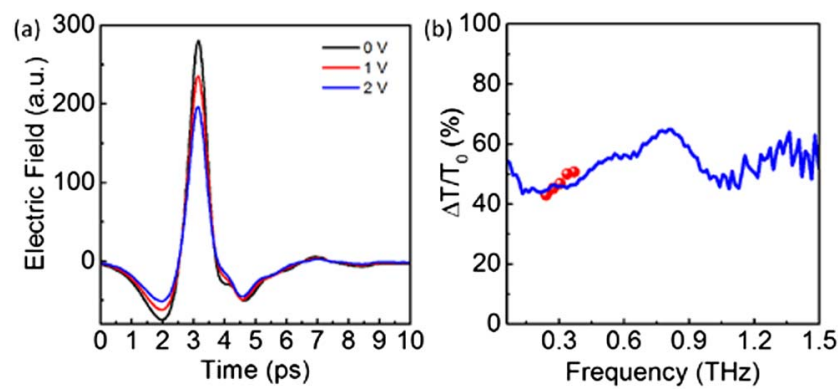

Fig. 3. (a) THz pulse recorded at bias voltages of 0,1 , and $2 \mathrm{~V}$. (b) Modulation plotted against frequency. The scattered points show the data recorded with our mm-wave/THz system.
The modulation varies between $40 \%$ and $60 \%$ in a frequency range of 0.1 to $1.5 \mathrm{THz}$. The scattered plot in Fig. 3(b) shows the modulation obtained from the mmwave/THz emitter system [Fig. 2(h)]. We observed a good agreement between the two measurement systems.

The ions of the electrolyte distribute themselves to screen the voltage of the graphene electrodes. Furthermore, the accumulated ions change the conductivity on the graphene electrodes via electrostatic doping. The charge accumulated on a pixel depends on not only the voltage difference between the top and bottom electrodes, but also the applied voltage on adjacent pixels. Figure 4(a) illustrates this process. The voltage difference between the adjacent pixels causes a cross talk. Due to the cross talk, the charge distribution (also the $\mathrm{THz}$ transmission) is not an ideal step-like function. Instead, the charge distribution changes gradually between the pixels. Figure 4(b) illustrates this complex charge distribution of a grounded bottom graphene electrode when the top graphene electrodes are biased at different voltages. Graphene shows ambipolar charge transport; therefore, the grounded electrode could be $n$-doped, $p$-doped, or neutral depending on the polarity of the voltage bias of the top electrode. Figure 4(c) shows measured THz transmittance along the center row of the image shown in Fig. 2(d). To quantify the cross talk, we defined a figure of merit as $\left(C=1-R_{\mathrm{av}} / R_{\max }\right)$ where $R_{\mathrm{av}} / R_{\max }$ is the ratio of averaged and maximum transmittance between two adjacent pixels. In the absence of cross talk, for example, the average and center values are the same, which yield $C=0$. In Fig. $\underline{4(\mathrm{~d})}$, we plotted the contrast $\left(T_{\max } / T_{\min }\right)$ and cross talk $(C=$ $\left.1-R_{\text {av }} / R_{\max }\right)$. We observe that there is a tradeoff between the contrast and the cross talk. The contrast increases with the bias voltage and saturates after a bias voltage of $2 \mathrm{~V}$ [Fig. 4(d)]. At the same time, cross talk increases with the bias voltage.

Finally, to understand the device performance in the high THz frequency range, a quantitative model based on a transfer matrix method for calculating the transmission coefficient of single-layer graphene was applied to
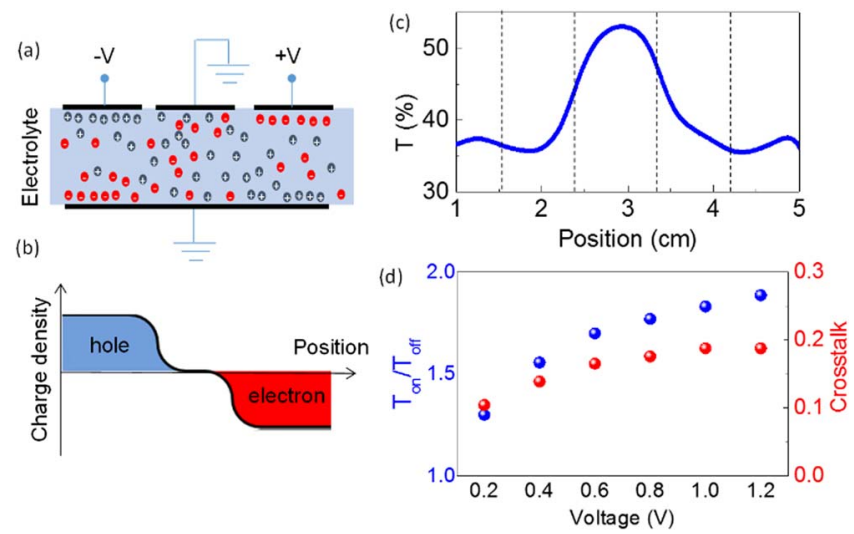

Fig. 4. (a) Schematic illustration of cross section of the device and (b) charge distribution on the graphene electrodes biased at different voltages. (c) Line profile of the $\mathrm{THz}$ transmission along the grounded row. (d) Variation of the modulation and cross talk of single pixel with bias voltage. 

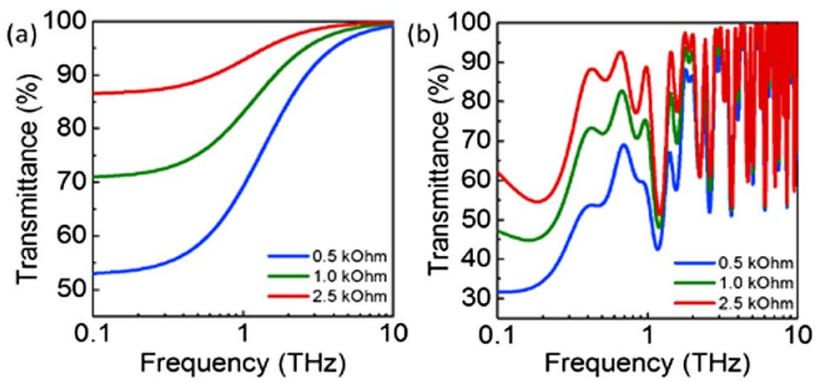

Fig. 5. (a) Calculated $\mathrm{THz}$ transmittance of single-layer graphene. (b) Simulated $\mathrm{THz}$ transmittance of graphene device.

our device [33,34]. We model the graphene electrodes as 0.3-nm-thick layers with Drude-like optical conductivity $\sigma(\omega)=\sigma_{\mathrm{DC}} /(1+i \omega \tau)$, where $\sigma_{\mathrm{DC}}$ is the low frequency conductivity of graphene, $\omega$ is the frequency, and $\tau$ is the mean scattering time of graphene electrodes, estimated to be about 200 fs. Figure 5(a) shows the calculated transmission of single-layer graphene with three different sheet resistances $\left(R_{s}=1 / \sigma\right)$. The modulation diminishes at $10 \mathrm{THz}$. These simulations suggest that our device can operate even in a broader frequency range. Using the measured values for the refractive index of the polymer substrate $\left(n_{s}=1.66,75 \mu \mathrm{m}\right.$ thick) and ionic liquid $\left(n_{\mathrm{L}}=2.31,80 \mu \mathrm{m}\right.$ thick), the overall modulator structure is simulated. Figure 5(b) shows the calculated $\mathrm{THz}$ transmittance spectrum of the device including the geometry of the device and the optical constant of the materials. The calculated modulation amplitude in the low $\mathrm{THz}$ frequency range agrees with the experiments.

In conclusion, we have demonstrated a broadband $\mathrm{THz}$ spatial light modulator using arrays of large area graphene supercapacitors. The THz transmittance of a single pixel is controlled using a passive matrix addressing. We anticipate that controlling $\mathrm{THz}$ waves by tuning high mobility carriers on large area CVD-grown graphene via efficient electrostatic gating would provide new opportunities to bridge the $\mathrm{THz}$ gap.

This work was supported by TUBITAK grant nos. 113F278 and 114F052. N. K. acknowledges the fellowship from TUBITAK-BIDEP.

\section{References}

1. M. Rahm, J. S. Li, and W. J. Padilla, J. Infrared Millimeter, Terahertz Waves 34, 1 (2013).

2. C. Sirtori, S. Barbieri, and R. Colombelli, Nat. Photonics 7, 691 (2013).

3. M. Tonouchi, Nat. Photonics 1, 97 (2007).

4. T. Kleine-Ostmann, P. Dawson, K. Pierz, G. Hein, and M. Koch, Appl. Phys. Lett. 84, 3555 (2004).

5. H. T. Chen, W. J. Padilla, J. M. O. Zide, A. C. Gossard, A. J. Taylor, and R. D. Averitt, Nature 444, 597 (2006).

6. H. T. Chen, W. J. Padilla, M. J. Cich, A. K. Azad, R. D. Averitt, and A. J. Taylor, Nat. Photonics 3, 148 (2009).

7. Y. Wu, X. Z. Ruan, C. H. Chen, Y. J. Shin, Y. Lee, J. Niu, J. B. Liu, Y. F. Chen, K. L. Yang, X. H. Zhang, J. H. Ahn, and H. Yang, Opt. Express 21, 21395 (2013).
8. W. L. Chan, H. T. Chen, A. J. Taylor, I. Brener, M. J. Cich, and D. M. Mittleman, Appl. Phys. Lett. 94, 213511 (2009).

9. Z. Xu and P. Mazumder, IEEE Trans. Electron Devices 61, 2195 (2014).

10. Z. W. Xie, X. K. Wang, J. S. Ye, S. F. Feng, W. F. Sun, T. Akalin, and Y. Zhang, Sci. Rep. 3, 3347 (2013).

11. S. Savo, D. Shrekenhamer, and W. J. Padilla, Adv. Opt. Mater. 2, 275 (2014).

12. D. Shrekenhamer, J. Montoya, S. Krishna, and W. J. Padilla, Adv. Opt. Mater. 1, 905 (2013).

13. S. Busch, B. Scherger, M. Scheller, and M. Koch, Opt. Lett. 37, 1391 (2012).

14. C. M. Watts, D. Shrekenhamer, J. Montoya, G. Lipworth, J. Hunt, T. Sleasman, S. Krishna, D. R. Smith, and W. J. Padilla, Nat. Photonics 8, 605 (2014).

15. M. Unlu, M. R. Hashemi, C. W. Berry, S. Li, S. H. Yang, and M. Jarrahi, Sci. Rep. 4, 5708 (2014).

16. L. J. Cheng and L. Liu, Opt. Express 21, 28657 (2013).

17. K. S. Novoselov, D. Jiang, F. Schedin, T. J. Booth, V. V. Khotkevich, S. V. Morozov, and A. K. Geim, Proc. Natl. Acad. Sci. USA 102, 10451 (2005).

18. B. Sensale-Rodriguez, T. Fang, R. S. Yan, M. M. Kelly, D. Jena, L. Liu, and H. L. Xing, Appl. Phys. Lett. 99, 113104 (2011).

19. P. Tassin, T. Koschny, and C. M. Soukoulis, Science 341, 620 (2013).

20. T. Low and P. Avouris, ACS Nano 8, 1086 (2014).

21. N. Rouhi, S. Capdevila, D. Jain, K. Zand, Y. Y. Wang, E. Brown, L. Jofre, and P. Burke, Nano Res. 5, 667 (2012).

22. T. Ando, A. B. Fowler, and F. Stern, Rev. Mod. Phys. 54, 437 (1982).

23. B. Sensale-Rodriguez, R. S. Yan, M. M. Kelly, T. Fang, K. Tahy, W. S. Hwang, D. Jena, L. Liu, and H. G. Xing, Nat. Commun. 3, 780 (2012).

24. B. Sensale-Rodriguez, S. Rafique, R. S. Yan, M. D. Zhu, V. Protasenko, D. Jena, L. Liu, and H. G. Xing, Opt. Express 21, 2324 (2013).

25. L. Ju, B. S. Geng, J. Horng, C. Girit, M. Martin, Z. Hao, H. A. Bechtel, X. G. Liang, A. Zettl, Y. R. Shen, and F. Wang, Nat. Nanotechnol. 6, 630 (2011).

26. R. Degl'Innocenti, D. S. Jessop, Y. D. Shah, J. Sibik, J. A. Zeitler, P. R. Kidambi, S. Hofmann, H. E. Beere, and D. A. Ritchie, Opt. Eng. 53, 057108 (2014).

27. A. Andryieuski and A. V. Lavrinenko, Opt. Express 21, 9144 (2013).

28. P. Y. Chen, H. Y. Huang, D. Akinwande, and A. Alu, ACS Photonics 1, 647 (2014).

29. W. L. Gao, J. Shu, K. Reichel, D. V. Nickel, X. W. He, G. Shi, R. Vajtai, P. M. Ajayan, J. Kono, D. M. Mittleman, and Q. F. $\mathrm{Xu}$, Nano Lett. 14, 1242 (2014).

30. B. Sensale-Rodriguez, R. S. Yan, S. Rafique, M. D. Zhu, W. Li, X. L. Liang, D. Gundlach, V. Protasenko, M. M. Kelly, D. Jena, L. Liu, and H. G. Xing, Nano Lett. 12, 4518 (2012).

31. E. O. Polat and C. Kocabas, Nano Lett. 13, 5851 (2013).

32. O. Balci, E. O. Polat, N. Kakenov, and C. Kocabas, Nat. Commun. 6, 6628 (2015).

33. W. D. Zhang, P. H. Q. Pham, E. R. Brown, and P. J. Burke, Nanoscale 6, 13895 (2014).

34. T. R. Zhan, X. Shi, Y. Y. Dai, X. H. Liu, and J. Zi, J. Phys. Condens. Matter 25, 215301 (2013). 